\title{
Strong association between herpes simplex virus- 1 and chemotherapy-induced oral mucositis in patients with hematologic malignancies
}

\author{
Junshik Hong ${ }^{1,2,}{ }^{*}$, Hee-Kyung Park ${ }^{3,4}{ }^{\star}$, Suhyun Park${ }^{4}$, Ahreum Lee ${ }^{5}$, Yeon-Hee Lee ${ }^{6}$, Dong-Yeop Shin ${ }^{1,2}$,
} Youngil $\mathrm{Koh}^{1,2}$, Ji-Yeob Choi ${ }^{2,7,8}$, Sung-Soo Yoon ${ }^{1,2}$, Youngnim $\mathrm{Choi}^{5}$, and Inho Kim ${ }^{1,2}$

\begin{abstract}
${ }^{1}$ Department of Internal Medicine, Seoul National University Hospital, Seoul; ${ }^{2}$ Cancer Research Institute, Seoul National University, Seoul; ${ }^{3}$ Department of Oral Medicine and Oral Diagnosis, Dental Research Institute, Seoul National University School of Dentistry, Seoul;

${ }^{4}$ Department of Oral Medicine, Seoul National University Dental Hospital, Seoul; ' 5 Department of Immunology and Molecular Microbiology, Dental Research Institute, Seoul National University School of Dentistry, Seoul; ${ }^{6}$ Department of Orofacial Pain and Oral Medicine, Kyung Hee University Dental Hospital, Seoul; ${ }^{7}$ Department of Preventive Medicine, Seoul National University College of Medicine, Seoul; ${ }^{8}$ Department of Biomedical Sciences, Seoul National University Graduate School, Seoul, Korea
\end{abstract}

Received: December 31, 2018

Revised : February 7, 2019

Accepted: March 25, 2019
Background/Aims: A link between oral cavity infections and chemotherapy-induced oral mucositis (CIOM) in patients with hematological malignancies (HMs) undergoing intensive chemotherapy (IC) or hematopoietic stem cell transplantation (HSCT) has been suggested. However, conclusive data are lacking, and there are no current guidelines for the prophylactic use of antimicrobials to prevent CIOM in these populations.

Methods: The relationships between herpes simplex virus (HSV) reactivation and Candida colonization in the oral cavity and CIOM in patients with HMs undergoing IC or HSCT were evaluated. Patients aged $\geq 19$ years with HMs undergoing IC or HSCT were enrolled. Each patient was evaluated for HSV and Candida in the oral cavity along with CIOM at baseline and during the $2 \mathrm{nd}$, $3 \mathrm{rd}$, and 4 th weeks.

Results: Seventy presentations among 56 patients were analyzed. CIOM was observed in 23 presentations (32.9\%), with a higher incidence associated with HSCT (17 of 35 presentations, 48.6\%) than with IC (six of 35 presentations, 8.6\%). The reactivation of HSV-1 was significantly associated with an increased incidence of CIOM after adjusting for age, sex, type of disease, and treatment stage. A higher HSV-1 viral load was associated with an increased incidence of CIOM. The presence of Candida was not associated with CIOM.

Conclusions: HSV-1 reactivation in the oral cavity was highly associated with CIOM in patients with HMs undergoing high-dose chemotherapy.

Keywords: Hematologic neoplasms; Stomatitis; Herpesvirus 1, human; Drug therapy; Stem cell transplantation

\section{Correspondence to Youngnim Choi, D.D.S.}

Department of Immunology and Molecular Microbiology, Dental Research Institute, Seoul National University School of Dentistry, 101 Daehak-ro, Jongno-gu, Seoul 0308o, Korea Tel: +82-2-740-8643, Fax:+82-2-745-1906, E-mail: youngnim@snu.ac.kr

Inho Kim, M.D.

Department of Internal Medicine, Seoul National University Hospital, 101 Daehak-ro, Jongno-gu, Seoul 03080, Korea

Tel: +82-2-2072-0834, Fax: +82-2-764-2199, E-mail: ihkimmd@snu.ac.kr

*These authors contributed equally to this work. 


\section{INTRODUCTION}

Antifolates, topoisomerase II inhibitors, high-dose alkylating agents, anthracyclines, and other anticancer drugs are known to be highly mucotoxic agents that can cause chemotherapy-induced oral mucositis (CIOM) [1]. Patients with hematological malignancies (HMs) are distinct from those with solid tumors because most HM patients receive high-dose, intensive chemotherapy (IC) that causes prolonged immunosuppression. Some patients with HMs may receive autologous or allogeneic hematopoietic stem cell transplantation (HSCT), which involves profound immunosuppression and various related complications. The incidence of CIOM among patients receiving HSCT is reportedly up to $70 \%[2-4]$, which is much higher than in patients who receive conventional chemotherapy [5]. These patients experience pain and functional disturbances such as dysphagia, leading to suboptimal nutritional status. CIOM may also delay or disrupt planned treatments and increase medical costs [6]. Most importantly, the CIOM-induced disruption of the oral mucosa provides a major route of entry for pathologic microorganisms, leading to various infections, including fatal septicemia $[7,8]$.

Both therapy- and patient-related risk factors influence the development of CIOM [9]. The dosage and duration of the exposure to chemotherapy influence CIOM development, as do therapy-related risk factors [1]. Patient-related factors include underlying comorbidities, malnutrition, trauma, and irritation caused by dentition, dental infection, and poor oral hygiene [1,9]. Microbial colonization has been proposed to be an exacerbating factor for oral mucositis and perhaps to even cause ulceration by itself [10]. Herpes simplex virus (HSV) [1113] and Candida spp. [11,12] have reportedly been associated with the incidence and severity of CIOM in patients with HM. For these reasons, the use of antimicrobials for the suppression of microbial colonization and the control of viral reactivation has been proposed $[8,14]$. However, previous studies showed inconclusive results regarding the role of antimicrobial prophylaxis for oral mucositis [10,15-17], and the Multinational Association of Supportive Care in Cancer guidelines that govern the management of mucositis secondary to cancer therapy do not specifically address CIOM prevention via the systemic use of antimicrobial agents [18]. Furthermore, the
Center for International Blood and Marrow Transplant Research (CIBMTR) guidelines limit the prophylactic use of acyclovir to HSV-seropositive allogeneic HSCT recipients [19]. The CIBMTR recommends administering acyclovir or valacyclovir during the pre-engraftment period to HSV-seropositive autologous HSCT recipients who are expected to experience severe CIOM; however, this recommendation is based only on expert opinion and is not widely supported [19].

The aim of the current study was to determine the prevalence of HSV reactivation and colonization with Candida, as well as the relationship between such oral microbial factors and CIOM development, in patients with HMs undergoing IC or HSCT.

\section{METHODS}

\section{Patients and study design}

The inclusion criteria for this study were (1) age $\geq 19$ years; (2) afflicted with an HM and admitted for IC or HSCT (either autologous or allogeneic); and (3) did not undergo or recover from any prior chemotherapy, radiation therapy, or surgery within the 3 weeks prior to enrollment. One patient with rhabdomyosarcoma who received autologous HSCT was included during their transplantation because they received an HM-equivalent treatment. Patients who satisfied all the inclusion criteria were eligible for enrollment multiple times. The exclusion criteria were (1) patients who already had definitive symptoms or signs of oral mucositis at baseline; (2) those who had other severe dental diseases or systemic diseases that could significantly affect the study outcomes, including active infection, uncontrolled diabetes, and symptomatic cardio- or cerebrovascular disorders; and (3) those who had underlying psychological diseases or cognitive disorders that precluded the necessary communication.

All enrolled patients received a basic oral and dental examination, as well as panoramic radiography when possible. The plaque index for periodontal diseases and the decayed, missing, and filled surface (DMFS) score [20] for dental caries were recorded within 2 days before or after the initiation of chemotherapy (baseline examinations). Evaluations of HSV-1, HSV-2, and Candida, and CIOM in the oral cavity were performed at baseline 

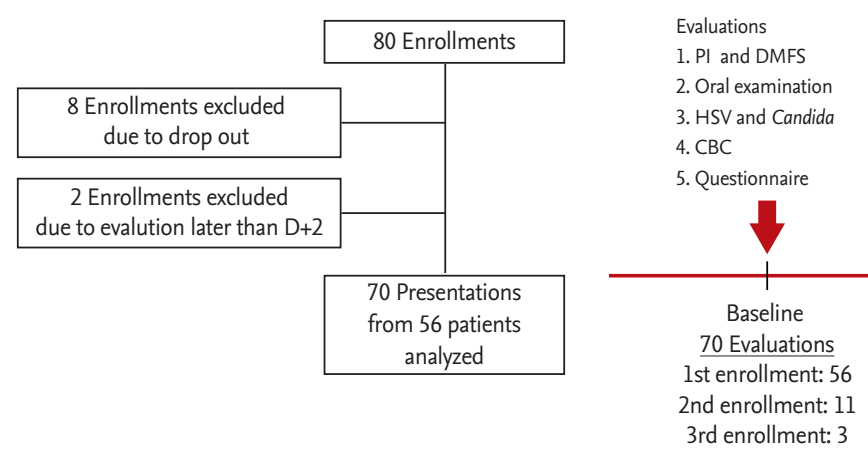

Figure 1. Flowchart of the study. PI, plaque index; DMFS, decayed, missing, and filled surface; HSV, herpes simplex virus; CBC, complete blood count.

(day $\mathrm{a} \pm 2$ ), week 2 (day $14 \pm 2$ ), week 3 (day $21 \pm 2$ ), and week 4 (day $28 \pm 2$ ), while the patients remained admitted (Fig. 1); missed appointments were documented. The patients were evaluated at least twice (at baseline and week 2) during each enrollment, or else their data were excluded from the analyses.

This cohort study was performed in accordance with the principles in the 1964 Declaration of Helsinki and its later amendments and was approved by the Institutional Review Board of Seoul National University School of Dentistry in Seoul, Korea (approval number: S-D20160016). Written informed consent was obtained from all patients as a condition of participation.

\section{Evaluation of HSV-1, HSV-2, and Candida in the oral cavity}

We defined HSV reactivation as the presence of the HSV genome in the oral mucosa. To evaluate the presence of HSV-1 and -2 in oral keratinocytes, a sample was obtained by placing a sterilized $30 \times 30 \mathrm{~mm}$ Immobilon-P Transfer Membrane (Merck Millipore, Billerica, MA, USA) on the buccal mucosa for 30 seconds. If CIOM developed, the sampling site included the CIOM lesions. DNA was isolated from the sampled membrane using a PowerSoil DNA Isolation Kit (MO BIO Laboratories, Carlsbad, CA, USA) and eluted in $100 \mathrm{~mL}$ elution buffer. In the preliminary experiment, the proper isolation of eukaryotic cell DNA was confirmed by the polymerase chain reaction (PCR) amplification of the $\beta$-actin gene fragment. The presence of HSV-1 and -2 was determined by PCR using an HSV 1/2 PCR kit (BioCore, Seoul, Korea). PCR mixtures were prepared by mixing $5 \mu \mathrm{L}$ reac- tion mixture, $3 \mu \mathrm{L}$ primer mix, and $2 \mu \mathrm{L}$ DNA samples, and amplification was performed under the following conditions: initial denaturation at $95^{\circ} \mathrm{C}$ for 12 mininutes; 35 cycles of $94^{\circ} \mathrm{C}$ for 45 seconds, $67^{\circ} \mathrm{C}$ for 45 seconds, and $72^{\circ} \mathrm{C}$ for 45 seconds; and finally, $72^{\circ} \mathrm{C}$ for 5 minutes. All PCRs were performed with positive, negative, and internal controls (Fig. 2). The viral loads of HSV-1 and -2 were first expressed as the ratios of the intensities of the HSV bands to the internal control bands, as measured by densitometry and categorized as $0,1(0<$ ratio $<$ median), and 2 (ratio $\geq$ median). Patients with positive results for the HSV PCR test were regarded as experiencing HSV reactivation.

To detect colonization with Candida, swabs of the buccal mucosa and the dorsum of the tongue of each patient were directly plated onto ChromID Candida Agar (BioMérieux, Lyon, France), which selects for yeasts and identifies Candida albicans. The plate was incubated at $37^{\circ} \mathrm{C}$ for 48 hours under aerobic conditions. The result was recorded as negative, carrier (less than 20 colony-forming units), or positive. To minimize the local effect of gargles, including nystatin, the swabs were performed while avoiding the time immediately after a meal and gargling.

Measures of infection prevention and applied oral care Prophylactic use of antibiotics, antifungal agents, and antiviral agents was decided according to the physicians' discretion and was mostly based on the reimbursement guidelines of the Korean Health Insurance Review and Assessment Service. Generally, patients undergoing IC received no prophylactic antibiotics, whereas ciproflox- 

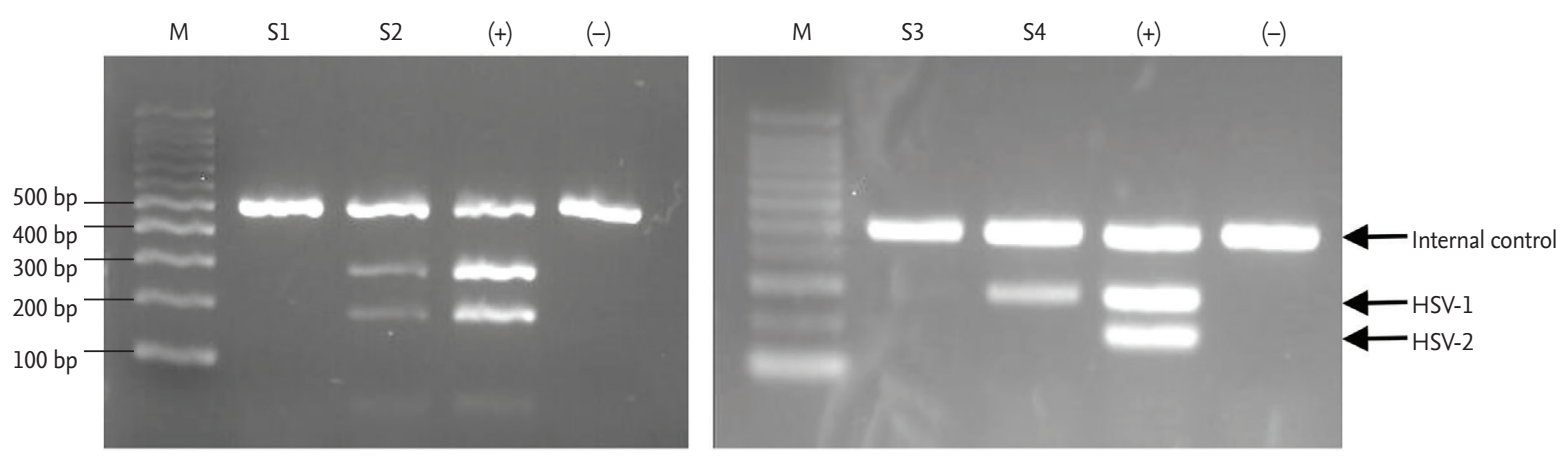

Figure 2. Polymerase chain reaction (PCR) for herpes simplex virus (HSV) 1 and 2. PCR was performed with a reaction mixture that included a template and primers for an internal control as well as primers for HSV-1 and -2. All PCR screenings of patient samples were carried out along with a positive sample provided in the kit (+) and water as a negative control (-).

acin (500 mg per os twice a day) was prescribed to HSCT recipients. Standard doses of posaconazole and micafungin were administered to patients with acute myeloid leukemia (AML)/myelodysplastic syndrome undergoing induction or reinduction chemotherapy and to HSCT recipients as antifungal prophylaxes. For patients receiving consolidative IC or in cases of other HMs such as acute lymphoblastic leukemia (ALL), antifungal prophylaxis was not routinely provided, although fluconazole or itraconazole was prescribed at the physician's discretion. In contrast, prophylactic anti-herpes virus drugs were not provided except to allogeneic HSCT recipients. All prophylactic antimicrobial agents were administered from the initiation of chemotherapy until neutrophil recovery, which was defined as an absolute neutrophil count $>1,000 / \mu \mathrm{L}$ for 3 consecutive days.

Patients were instructed to rinse their oral cavities with normal saline four times daily and with chlorhexidine twice daily. To prevent oral candidiasis, patients gargled with 5 to $10 \mathrm{~mL}$ of nystatin oral suspension three times daily right after a meal during the study period. Standard infection prevention measures that were applied, including isolation with a high efficiency particulate air-filtered laminar flow hood, low bacterial diet during the neutrophil count nadir, hand-washing and hygiene practices, such as wearing surgical masks when contacting personnel.

\section{Evaluation of CIOM}

At every evaluation (baseline, week 2, 3, and 4), the CIOM presence was estimated and graded according to the World Health Organization (WHO) oral toxicity scale
[21] and the National Cancer Institute Common Toxicity Criteria for Adverse Events (NCI-CTCAE), version 3.O. Among the oral lesions, typical HSV-associated vesicular lesions and HSV-associated ulcers were not considered CIOM. HSV-associated ulcers, which occur in nonmovable keratinized gingiva (attached gingiva, hard palate, and tongue dorsum), were differentiated from mucositis-related ulcers, which occur in movable mucosa (buccal mucosa, soft palate, labial mucosa, and ventral tongue). At every evaluation, patients completed the Oral Mucositis Daily Questionnaire (OMDQ), which was identical to that used previously except for the exclusion of two diarrhea-related questions [22].

\section{Review of medical records}

Baseline characteristics of the patients, such as age, sex, and diagnosis, were recorded. Stages of IC or HSCT for the enrolled patients were classified as (1) induction, (2) consolidation, (3) reinduction or salvage, and (4) HSCT. Although the treatment regimens were heterogeneous among patients, the induction regimens for AML and ALL were mostly cytarabine plus anthracycline (so-called $7+$ 3) and vincristine, prednisolone, daunorubicin, and L-asparaginase (VPDL), respectively. Both myeloablative and reduced-intensity conditioning with or without antithymocyte globulin were allowed. However, no patient with a total body irradiation-integrated conditioning regimen was included. Any use of antibiotics, antifungal agents, and antiviral agents from day -7 to 28 was recorded.

\section{Statistical analysis}

The incidence of CIOM was calculated based on en- 
Table 1. Patient characteristics and the relationship of the patient characteristics to the incidence of CIOM

\begin{tabular}{|c|c|c|c|c|c|c|}
\hline \multirow[t]{2}{*}{ Characteristic } & \multicolumn{3}{|c|}{ Patient number ${ }^{\mathrm{a}}$} & \multicolumn{3}{|c|}{$\begin{array}{l}\text { CIOM according to the WHO/NCI-CT- } \\
\text { CAE version } 3.0 \text { (yes vs. no) }\end{array}$} \\
\hline & Total & With CIOM & Without CIOM & Odds ratio $^{c}$ & $95 \% \mathrm{CI}^{\mathrm{c}}$ & $p$ value ${ }^{c}$ \\
\hline \multicolumn{7}{|l|}{ Sex } \\
\hline Male & 34 & 14 & 20 & Reference & & \\
\hline Female & 36 & 9 & 27 & 0.221 & $0.093-0.525$ & 0.001 \\
\hline Age, median, yr & $53 \cdot 5$ & 53.0 & $57 \cdot 0$ & 0.996 & $0.961-1.033$ & 0.847 \\
\hline \multicolumn{7}{|l|}{ Type of disease } \\
\hline Acute leukemia or MDS & 46 & 11 & 35 & Reference & & \\
\hline Multiple myeloma & 15 & 7 & 8 & 0.655 & $0.205-2.092$ & 0.475 \\
\hline Lymphoma & 8 & 5 & 3 & 0.728 & $0.214-2.472$ & 0.611 \\
\hline Other: rhabdomyosarcoma & 1 & o & 1 & - & & \\
\hline \multicolumn{7}{|l|}{ Treatment stage } \\
\hline Induction chemotherapy & 18 & 5 & 13 & Reference & - & - \\
\hline Consolidative chemotherapy & 13 & 1 & 12 & 0.168 & $0.019-1.515$ & 0.112 \\
\hline Reinduction or salvage & 4 & 0 & 4 & - & - & - \\
\hline HSCT & 35 & 17 & 18 & 5.900 & $1.606-21.670$ & 0.007 \\
\hline Autologous & 23 & 12 & 11 & & & \\
\hline Allogeneic & 12 & 5 & 7 & & & \\
\hline Plaque index, median (range) & $0.53(0-2.13)$ & $0.42(0.1-1.33)$ & $0.29(0-2.13)$ & 0.574 & $0.164-2.006$ & 0.385 \\
\hline DMFS score, median (range) & $15.0(0-51.7)$ & $11.4(3 \cdot 3-35)$ & $16.1(0-51.7)$ & 0.970 & $0.926-1.016$ & 0.201 \\
\hline
\end{tabular}

CIOM, chemotherapy-induced oral mucositis; WHO, World Health Organization; NCI-CTCAE, National Cancer Institute-Common Toxicity Criteria for Adverse Events; CI, confidence interval; MDS, myelodysplastic syndrome; HSCT, hematopoietic stem cell transplantation; DMFS, Decayed, Missed, Filled Surface.

${ }^{a}$ Among 70 presentations.

${ }^{\mathrm{b}}$ According to multiple logistic regression analysis among 224 evaluations.

${ }^{\mathrm{c}}$ Adjusted for age, sex, type of disease, and treatment stage.

rollment. Since multiple evaluations were performed during each enrollment, relationships between various factors, including patient characteristics, microbial factors, and OMDQ data, were analyzed from among the 224 evaluations. Odds ratios and $95 \%$ confidence intervals for the associations of explanatory variables with binary outcomes were estimated by multiple logistic regression models, and coefficients and $p$ values for the explanatory variables with continuous outcomes were estimated by linear regression models. After independent variables were screened as possible explanatory variables for CIOM, age, sex, type of disease, and treatment stage were adjusted as possible confounders in the final model to test the association of HSV and Candi$d a$ with CIOM. $p$ values $<0.05$ were considered statistically significant. Tests were two-sided, and all statisti- cal analyses were calculated using STATA version 12.0 (StataCorp., College Station, TX, USA).

\section{RESULTS}

\section{Patient enrollment and treatment}

Patients were enrolled between July 2016 and May 2017. Initially, 8o patients were enrolled; however, 10 were subsequently excluded (Fig. 1). Because eight patients were enrolled twice and three were enrolled thrice, 70 presentations from 56 patients were finally analyzed. Overall, 224 oral and dental evaluations were conducted for the 70 presentations during the study period (Fig. 1). The characteristics of the enrolled patients are summarized in Table 1. Of the 70 presentations, antibiotics 


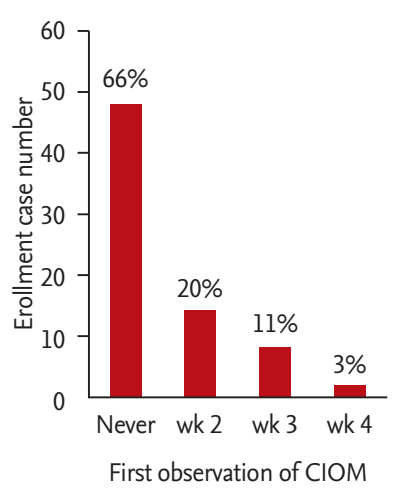

A

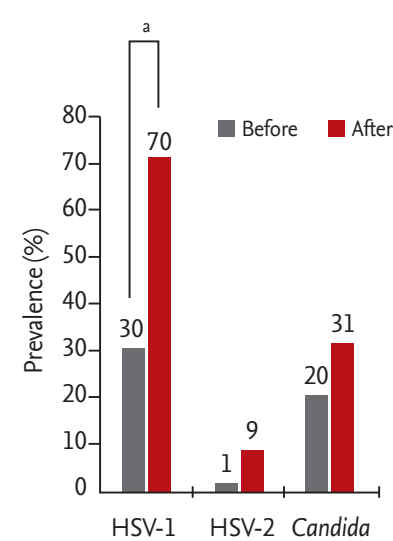

B

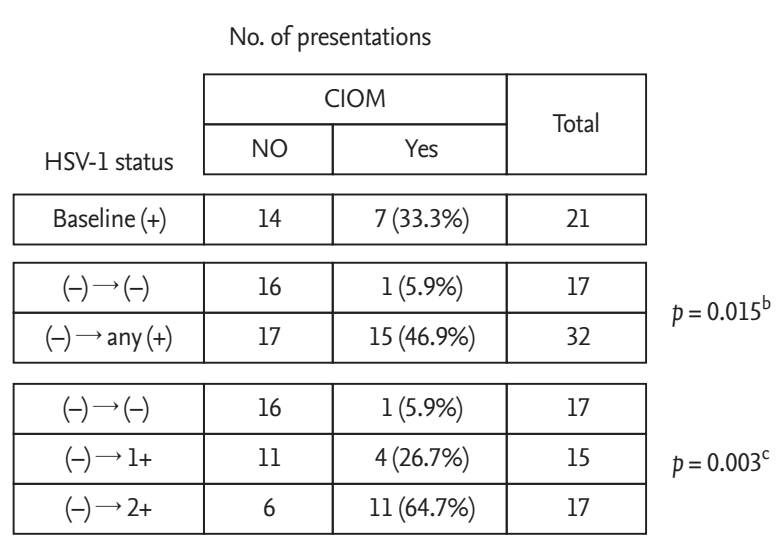

bBetween two groups, according to chi-square test

'Between three groups, according to chi-square test

Figure 3. Evaluation of chemotherapy-induced oral mucositis (CIOM) and the presence of herpes simplex virus (HSV) in the oral cavity. (A) The first observation of CIOM during the periods of evaluation. (B) Prevalence of HSV-1, HSV-2, and Candida before and after treatment. (C) Analysis of the association between HSV-1 status and the occurrence of CIOM after excluding patients who showed HSV-1 at baseline examination. ${ }^{\mathrm{a}} \mathrm{p}<0.05$, ${ }^{\mathrm{b}}$ Between two groups, according to chi-square test, ${ }^{\mathrm{c}} \mathrm{Between}$ three groups, according to chi-square test.

were used from the initiation of chemotherapy in 56. Antifungal and antiviral prophylaxes were applied in 60 and 12 (acyclovir for only the allogeneic HSCT recipients) presentations, respectively.

\section{Incidences of $\mathrm{CIOM}$ and the associated patient characteristics}

Among the 70 presentations, 23 (32.9\%) experienced CIOM, which was first observed in week 2 in most cases, although some cases were first observed in week 3 or 4 (Fig. 3A). Patients with HSCT showed a higher incidence of CIOM ( 17 of 35 presentations [48.6\%]; 12/23 and 5/12 for autologous and allogeneic HSCT, respectively) than that of patients with IC (six of 35 presentations [8.6\%]; 5/18, $1 / 13$, and $\mathrm{o} / 4$ for induction, consolidation, and reinduction, respectively).

The incidences of CIOMs that were estimated according to the WHO and NCI-CTCAE version 3.0 scales were highly correlated; both scales showed 100\% agreement regarding the incidence of CIOM (yes vs. no) for the 33 positive evaluations among the 224 total evaluations. Male sex and HSCT were associated with an increased incidence of CIOM that was estimated by the WHO (and NCI-CTCAE version 3.0) scale. The plaque index and DMFS score were not associated with the incidence of CIOM (Table 1).

\section{Microbial factors associated with CIOM}

Among the 224 evaluations, HSV-1 was detected in 94 evaluations (42.0\%; 46 and 48 for viral-load categories 1 and 2, respectively). HSV-2 was detected only in seven evaluations (3.1\%; four and three for categories 1 and 2, respectively), and all cases except for one case that was observed at baseline were detected together with HSV1. The prevalence of HSV and Candida was increased compared to that at baseline after IC or HSCT, but the increase was only significant for HSV-1 (Fig. 3B). The reactivation of HSV-1 was significantly associated with an increased incidence of CIOM after the data were adjusted for age, sex, type of disease, and treatment stage (Table 2). In contrast, the detection of either HSV-2 or Candida was not associated with CIOM (Table 2). To exclude a bystander infection of HSV-1, the association of HSV1 with CIOM was further analyzed after excluding the presentations that had HSV-1 reactivation at baseline (Fig. 3 C). Patients with newly detected HSV-1 in the oral cavity during treatment showed a significantly higher incidence of CIOM $(15 / 32=46.9 \%)$ compared to that of patients who did not have HSV-1 during treatment $(1 / 17$ $=5.9 \%)$. Eleven out of the 17 patients (64.7\%) with a high HSV-1 viral load (2+) developed CIOM, whereas did four out of the 15 patients $(26.7 \%)$ with a low HSV-1 viral load (1+) suggesting that there was a relationship between the 
Table 2. Relationship between the presence of HSV-1 and -2 and the incidence of CIOM

\begin{tabular}{|c|c|c|c|}
\hline \multirow{2}{*}{ Category of viral load (no. of evaluations) } & \multicolumn{3}{|c|}{ CIOM according to the WHO/NCI-CTCAE version 3.0 (yes vs. no) } \\
\hline & Odds ratio $^{\mathrm{b}}$ & $95 \% \mathrm{CI}^{\mathrm{b}}$ & $p$ value $^{\mathrm{b}}$ \\
\hline \multicolumn{4}{|l|}{ HSV-1 } \\
\hline$o(n=130)$ & Reference & & \\
\hline $1(n=46)$ & 3.693 & $1.032-13.213$ & 0.045 \\
\hline $2(n=48)$ & 16.913 & $3.922-72.929$ & $<0.001$ \\
\hline $1+2(n=94)$ & 7.660 & $2.762-21.242$ & $<0.001$ \\
\hline \multicolumn{4}{|l|}{$\mathrm{HSV}-2$} \\
\hline $\mathrm{o}(\mathrm{n}=217)$ & Reference & & \\
\hline $1+2(n=7)$ & $13 \cdot 370$ & $0.962-185.727$ & 0.053 \\
\hline \multicolumn{4}{|l|}{ Candida } \\
\hline Negative $(n=184)$ & Reference & & \\
\hline Carrier $(\mathrm{n}=20)$ & 0.591 & $0.129-2.716$ & 0.499 \\
\hline Positive $(\mathrm{n}=17)$ & 0.435 & $0.045-4.161$ & 0.470 \\
\hline
\end{tabular}

HSV, herpes simplex virus; CIOM, chemotherapy-induced oral mucositis; WHO, World Health Organization; NCI-CTCAE, National Cancer Institute-Common Toxicity Criteria for Adverse Events; CI, confidence interval.

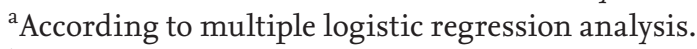

${ }^{\mathrm{b}}$ Adjusted for age, sex, type of disease, and treatment stage.

Table 3. Relationship between oral/throat pain and the incidence of CIOM

\begin{tabular}{|c|c|c|c|c|}
\hline \multirow{2}{*}{\multicolumn{2}{|c|}{$\begin{array}{l}\text { Questions for oral/throat pain according to the } \\
\text { OMDQ (scales) }\end{array}$}} & \multicolumn{3}{|c|}{ CIOM according to the WHO/NCI-CTCAE version 3.0 (yes vs. no) } \\
\hline & & \multirow{2}{*}{\multicolumn{3}{|c|}{$\begin{array}{ccr}\beta & 95 \% \text { CI } & p \text { value } \\
1.153 & -0.246 \text { to } 2.552 & 0.106\end{array}$}} \\
\hline Q.1 & Overall health status (o, worst-10, best) & & & \\
\hline Q.2 & Pain in mouth and throat (o, none-4, severe) & 1.531 & 1.268 to 1.795 & $<0.001$ \\
\hline Q.3a & Limitation of swallowing (o, none-4, severe) & 0.894 & 0.660 to 1.129 & $<0.001$ \\
\hline Q.3b & Limitation of drinking water (o, none-4, severe) & 0.879 & 0.629 to 1.129 & $<0.001$ \\
\hline Q.3C & Limitation of eating meals (o, none-4, severe) & 1.488 & 1.185 to 1.791 & $<0.001$ \\
\hline Q.3d & Limitation of speech (o, none-4, severe) & 0.774 & 0.584 to 0.964 & $<0.001$ \\
\hline Q.3e & Limitation of sleeping (o, none-4, severe) & 0.488 & 0.276 to 0.700 & $<0.001$ \\
\hline Q.4 & Overall oral and throat pain (o, none-10, severe) & $4 \cdot 514$ & 3.753 to 5.275 & $<0.001$ \\
\hline
\end{tabular}

CIOM, chemotherapy-induced oral mucositis; OMDQ, Oral Mucositis Daily Questionnaire; WHO, World Health Organization; NCI-CTCAE, National Cancer Institute-Common Toxicity Criteria for Adverse Events; CI, confidence interval.

${ }^{a}$ According to multiple linear regression analysis (reference: no CIOM).

HSV-1 viral load and the occurrence of CIOM.

\section{Relationship between subjective patient complaints and CIOM or the reactivation of HSV}

We investigated the relationship between the severity of the subjective complaints of oral pain or of the limitation of function (measured by the OMDQ) and the incidence of CIOM. As the patients' complaints of oral pain or of the limitation of oral and dental function became more severe, an increased incidence of CIOM was observed (Table 3). Intriguingly, these results were not associated with the overall health status (question no. 1 on the OMDQ). The OMDQ scores were also associated with HSV-1 reactivation and the viral load category (Table 4). 
Table 4. Relationship between oral/throat pain and the presence of HSV-1 and viral load across all the evaluations

\begin{tabular}{|c|c|c|c|c|c|c|}
\hline \multirow{2}{*}{\multicolumn{4}{|c|}{ Questions for oral/throat pain according to the OMDQ (scales) }} & \multicolumn{3}{|c|}{ 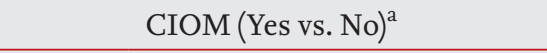 } \\
\hline & & & & \multirow{2}{*}{$\begin{array}{c}\beta \\
0.697 \\
0.635 \\
0.758\end{array}$} & \multirow{2}{*}{$\begin{array}{c}95 \% \text { CI } \\
-0.245 \text { to } 1.639 \\
-0.551 \text { to } 1.820 \\
-0.444 \text { to } 1.961\end{array}$} & \multirow{2}{*}{$\begin{array}{c}\text { p value } \\
0.147 \\
0.294 \\
0.217\end{array}$} \\
\hline Q.1 & $\begin{array}{l}\text { Overall health status } \\
\text { (o, worst-10, best) }\end{array}$ & $\begin{array}{l}\text { HSV-1 reactivation } \\
\text { HSV-1 viral load }\end{array}$ & $\begin{array}{l}\text { Yes vs. No } \\
1 \text { vs. } 0 \\
2 \text { vs. } O\end{array}$ & & & \\
\hline Q.2 & $\begin{array}{l}\text { Pain in mouth and throat } \\
\text { (o, no- } 4 \text {, severe) }\end{array}$ & $\begin{array}{l}\text { HSV-1 reactivation } \\
\text { HSV-1 viral load }\end{array}$ & $\begin{array}{l}\text { Yes vs. No } \\
1 \text { vs. } 0 \\
2 \text { vs. } 0\end{array}$ & $\begin{array}{l}0.463 \\
0.290 \\
0.642\end{array}$ & $\begin{array}{l}0.243 \text { to } 0.683 \\
0.016 \text { to } 0.564 \\
0.365 \text { to } 0.919\end{array}$ & $\begin{array}{r}<0.001 \\
0.038 \\
<0.001\end{array}$ \\
\hline Q.3a & $\begin{array}{l}\text { Limitation of swallowing } \\
(0, \text { no-4, severe })\end{array}$ & $\begin{array}{l}\text { HSV-1 reactivation } \\
\text { HSV-1 viral load }\end{array}$ & $\begin{array}{l}\text { Yes vs. No } \\
1 \text { vs. } 0 \\
2 \text { vs. } 0\end{array}$ & $\begin{array}{l}0.216 \\
0.148 \\
0.285\end{array}$ & $\begin{array}{r}0.037 \text { to } 0.395 \\
-0.077 \text { to } 0.373 \\
0.059 \text { to } 0.511\end{array}$ & $\begin{array}{l}0.018 \\
0.197 \\
0.013\end{array}$ \\
\hline Q.3b & $\begin{array}{l}\text { Limitation of drinking water } \\
\text { (o, no-4, severe) }\end{array}$ & $\begin{array}{l}\text { HSV-1 reactivation } \\
\text { HSV-1 viral load }\end{array}$ & $\begin{array}{l}\text { Yes vs. No } \\
1 \text { vs. } 0 \\
2 \text { vs. } 0\end{array}$ & $\begin{array}{l}0.194 \\
0.136 \\
0.253\end{array}$ & $\begin{array}{r}0.006 \text { to } 0.382 \\
-0.101 \text { to } 0.372 \\
0.015 \text { to } 0.491\end{array}$ & $\begin{array}{l}0.043 \\
0.261 \\
0.037\end{array}$ \\
\hline Q.3C & $\begin{array}{l}\text { Limitation of eating meals } \\
\text { (o, no-4, severe) }\end{array}$ & $\begin{array}{l}\text { HSV-1 reactivation } \\
\text { HSV-1 viral load }\end{array}$ & $\begin{array}{l}\text { Yes vs. No } \\
1 \text { vs. } 0 \\
2 \text { vs. } 0\end{array}$ & $\begin{array}{l}0.376 \\
0.259 \\
0.496\end{array}$ & $\begin{array}{r}0.133 \text { to } 0.620 \\
-0.047 \text { to } 0.564 \\
0.189 \text { to } 0.804\end{array}$ & $\begin{array}{l}0.002 \\
0.097 \\
0.002\end{array}$ \\
\hline Q.3d & $\begin{array}{l}\text { Limitation of speech } \\
\text { (o, no-4, severe) }\end{array}$ & $\begin{array}{l}\text { HSV-1 reactivation } \\
\text { HSV-1 viral load }\end{array}$ & $\begin{array}{l}\text { Yes vs. No } \\
1 \text { vs. } 0 \\
2 \text { vs. } 0\end{array}$ & $\begin{array}{l}0.210 \\
0.108 \\
0.313\end{array}$ & $\begin{array}{r}0.063 \text { to } 0.356 \\
-0.075 \text { to } 0.291 \\
0.129 \text { to } 0.497\end{array}$ & $\begin{array}{l}0.005 \\
0.246 \\
0.001\end{array}$ \\
\hline Q.3e & $\begin{array}{l}\text { Limitation of sleeping } \\
\text { (o, no-4, severe) }\end{array}$ & $\begin{array}{l}\text { HSV-1 reactivation } \\
\text { HSV-1 viral load }\end{array}$ & $\begin{array}{l}\text { Yes vs. No } \\
1 \text { vs. } 0 \\
2 \text { vs. } O\end{array}$ & $\begin{array}{l}0.074 \\
0.025 \\
0.128\end{array}$ & $\begin{array}{l}-0.077 \text { to } 0.226 \\
-0.165 \text { to } 0.215 \\
-0.065 \text { to } 0.322\end{array}$ & $\begin{array}{l}0.337 \\
0.798 \\
0.194\end{array}$ \\
\hline Q.4 & $\begin{array}{l}\text { Overall oral and throat pain } \\
\text { (o, no-10, severe) }\end{array}$ & $\begin{array}{l}\text { HSV-1 reactivation } \\
\text { HSV-1 viral load }\end{array}$ & $\begin{array}{l}\text { Yes vs. No } \\
1 \text { vs. } 0 \\
2 \text { vs. } 0\end{array}$ & $\begin{array}{l}1.511 \\
0.809 \\
2.226\end{array}$ & $\begin{array}{c}0.879 \text { to } 2.143 \\
0.028 \text { to } 1.589 \\
1.438 \text { to } 3.013\end{array}$ & $\begin{array}{r}<0.001 \\
0.042 \\
<0.001\end{array}$ \\
\hline
\end{tabular}

HSV, herpes simplex virus; OMDQ, Oral Mucositis Daily Questionnaire; CIOM, chemotherapy-induced oral mucositis; CI, confidence interval.

${ }^{\mathrm{a} A c c o r d i n g ~ t o ~ m u l t i p l e ~ l i n e a r ~ r e g r e s s i o n ~ a n a l y s i s . ~}$

\section{DISCUSSION}

We found a strong association between HSV-1 reactivation in the oral cavity and CIOM in patients with HMs. Only one of the 23 presentations involving CIOM did not experience HSV reactivation during the evaluation period. Furthermore, HSV-1 reactivation showed the highest odds ratio among the examined covariates, even after adjusting for other known factors (Table 2).

Several groups investigated a potential role for HSV in CIOM in the 1990s, but most of the groups reported negative results [15-17]. In a study of 60 HSCT recipients [17], all except one of whom were administered prophylactic acyclovir, Woo et al. [17] found that 45 of the 47 patients who developed ulcerative lesions (CIOM) were culture-negative for HSV. These researchers conclud- ed that HSV was probably not a major etiologic factor for CIOM in HSCT recipients [17]. Earlier studies also found that HSV plays an insignificant role or is only one of many cofactors that affect CIOM in the settings of HMs that are treated with IC $[15,16]$, solid tumors that are treated with chemo/radiation therapy [23], and pediatric cancers [24]. However, one study showed an association between the presence of HSV in the saliva and severe intraoral ulcers [25]. More recent studies [11,12,26] have revealed a relationship between HSV and CIOM; however, the present study identified HSV-1 as a risk factor for CIOM that was independent of age, sex, treatment stage, and type of disease. The reason for the difference in the results of earlier studies (i.e., no relationship of HSV to CIOM) [15-17] versus more recent studies (i.e., a strong association of HSV with CIOM) [11,12,26] could 
be attributed to the more frequent use of antimicrobial agents in more recent days [27]. Because antibiotics or antifungal agents are so commonly used either prophylactically or therapeutically, it is more difficult for bacteria or fungi to cause CIOM. In contrast, the use of antiviral agents is relatively limited compared to that of antibacterial or antifungal agents; thus, HSV could be the current main causative microorganism for CIOM. In line with this hypothesis, Chen et al. [11] reported that the association between HSV-1 infection and CIOM was significantly higher in patients who had received antifungal agents prior to CIOM occurrence than that in patients who had not received antifungal agents. Another potential explanation is that the HSV detection technology has improved. We and others [12,26] used a PCRbased method that is more sensitive for HSV detection [28] than immunofluorescence- [15,17] or culture-based $[16,25]$ methods.

Notably, 27 presentations did not develop CIOM despite $\mathrm{HSV}_{-1}$ reactivation, suggesting that additional factors such as mucosal injury by mastication may be required for CIOM development. Some patients reportedly experience oral/throat soreness due to HSV-1 reactivation-induced nerve sensitization, although overt inflammation (i.e., CIOM) does not necessarily occur [29]. Nevertheless, these data illustrate the importance of controlling HSV-1 in patients with HMs undergoing IC or HSCT.

The incidence rate of CIOM was $48.6 \%$ in the HSCT setting; while this rate is high, it is somewhat lower than that in previous studies [2-4], possibly because allogeneic HSCT recipients with nonmyeloablative conditioning were included. The incidence rate of CIOM was $22.7 \%$ in patients receiving induction or reinduction chemotherapy but was only $7.7 \%$ in those undergoing consolidative chemotherapy. These data suggest that the incidence of CIOM is substantially lower in patients receiving consolidative IC and that HSCT recipients and patients undergoing induction chemotherapy should be considered at high risk for developing CIOM.

The patients' reports of pain were highly correlated with the incidence of CIOM; hence, patient-reported questionnaires can be integrated into clinical practice for CIOM screening. The lack of an association of the overall health status with CIOM suggests that oral and dental problems should be considered independently rather than as a reflection of the general and/or systemic adverse effects of IC or HSCT.

This study had limitations. The roles of antimicrobial agents in the occurrence of CIOM were not thoroughly evaluated because all three kinds of antimicrobials were used frequently for different purposes and with variable doses, durations, and routes; hence, it was impossible to identify specific associations. However, since we followed routine clinical practices, our results reflect more real-life situations related to HM treatment. Second, we did not determine the status of the anti-HSV immunoglobulin G antibody before IC or HSCT, as we assumed that almost all patients carry the antibody, based on a previous study that found a $100 \% \mathrm{HSV}-1$ seropositive rate in Koreans aged $\geq 30$ years [30]. Third, we defined 'HSV reactivation' as the presence of the HSV genome in the oral mucosa. HSV reactivation was not functionally validated by other methods, such as viral culture or a histopathologic examination for the formation of inclusion bodies. Since the seropositive status of patients at baseline was not confirmed, one may argue that some cases could be a primary HSV infection rather than a reactivation. However, we regarded all cases that were positive for the HSV PCR test as a 'reactivation' because there were no patients who showed the clinical features of primary HSV infection and in consideration of the high seropositive rate for HSV-1 in Korean patients. Fourth, the chemotherapeutic and conditioning regimens among the patients varied, although we analyzed the association of HSV-1 with CIOM incidence after adjusting for the patients' treatment stage. Finally, although we swabbed the oral cavity of patients while avoiding the time immediately after a meal and gargling, some local effects of gargles, including nystatin, as well as systemic anti-fungal prophylaxis, might have had an impact on the lack of the association between oral candidiasis and CIOM in some patients.

Because we designed the present study with a maximum of four intermittent timepoints for evaluation during each treatment course, we could not estimate the serial and chronological changes of CIOM (i.e., the onset, changes of severity, and resolution of CIOM) and other variables. Notwithstanding, the design of our study enabled the evaluation of the true association between HSV-1 reactivation and the occurrence of CIOM at each timepoint. 
In conclusion, $\mathrm{HSV}-1$ reactivation in the oral cavity was highly associated with the incidence of CIOM in patients with HM undergoing IC or HSCT. A prospective trial is therefore warranted to evaluate the effect of prophylactic acyclovir on CIOM prevention in HM patients receiving induction chemotherapy or autologous HSCT. In addition, six of the 12 patients who underwent allogeneic HSCT presented HSV-1 despite the use of prophylactic anti-herpes viral agents. Therefore, the effective dose for the prevention of HSV-related CIOM needs to be re-evaluated.

\section{KEY MESSAGE}

1. The incidence rate of chemotherapy-induced oral mucositis (CIOM) was $32.9 \%$ in patients with hematologic malignancies undergoing high-dose chemotherapy, with a higher incidence rate associated with stem cell transplantation $(48.6 \%)$ than with intensive chemotherapy (8.6\%).

2. Herpes simplex virus 1 (HSV-1) reactivation in the oral cavity was highly associated with CIOM in patients with hematological malignancies undergoing high-dose chemotherapy. A high HSV-1 viral load was associated with an increased incidence of CIOM.

\section{Conflict of interest}

No potential conflict of interest relevant to this article was reported.

\section{Acknowledgments}

This research was supported by the Mid-Career Researcher Program of the National Research Foundation funded by the Ministry of Science \& ICT, Republic of Korea (2017R1A2B4002176) and the Convergence Research Program from the School of Dentistry and College of Medicine, Seoul National University (86020160116, 800-20160461).

\section{REFERENCES}

1. Sonis ST, Elting LS, Keefe D, et al. Perspectives on cancer therapy-induced mucosal injury: pathogenesis, measurement, epidemiology, and consequences for patients. Cancer 2004;100:1995-2025.

2. Vera-Llonch M, Oster G, Ford CM, Lu J, Sonis S. Oral mucositis and outcomes of allogeneic hematopoietic stem-cell transplantation in patients with hematologic malignancies. Support Care Cancer 2007;15:491-496.

3. Vokurka S, Bystricka E, Koza V, et al. Higher incidence of chemotherapy induced oral mucositis in females: a supplement of multivariate analysis to a randomized multicentre study. Support Care Cancer 2006;14:974-976.

4. Vagliano L, Feraut C, Gobetto G, et al. Incidence and severity of oral mucositis in patients undergoing haematopoietic SCT: results of a multicentre study. Bone Marrow Transplant 2011;46:727-732.

5. Jones JA, Avritscher EB, Cooksley CD, Michelet M, Bekele BN, Elting LS. Epidemiology of treatment-associated mucosal injury after treatment with newer regimens for lymphoma, breast, lung, or colorectal cancer. Support Care Cancer 2006;14:505-515.

6. Sonis ST, Oster G, Fuchs H, et al. Oral mucositis and the clinical and economic outcomes of hematopoietic stemcell transplantation. J Clin Oncol 2001;19:2201-2205.

7. Ruescher TJ, Sodeifi A, Scrivani SJ, Kaban LB, Sonis ST. The impact of mucositis on alpha-hemolytic streptococcal infection in patients undergoing autologous bone marrow transplantation for hematologic malignancies. Cancer 1998;82:2275-2281.

8. Scully C, Sonis S, Diz PD. Oral mucositis. Oral Dis 2006; 12:229-241.

9. Pico JL, Avila-Garavito A, Naccache P. Mucositis: its occurrence, consequences, and treatment in the oncology setting. Oncologist 1998;3:446-451.

10. Donnelly JP, Bellm LA, Epstein JB, Sonis ST, Symonds RP. Antimicrobial therapy to prevent or treat oral mucositis. Lancet Infect Dis 2003;3:405-412.

11. Chen YK, Hou HA, Chow JM, Chen YC, Hsueh PR, Tien HF. The impact of oral herpes simplex virus infection and candidiasis on chemotherapy-induced oral mucositis among patients with hematological malignancies. Eur J Clin Microbiol Infect Dis 2011;30:753-759.

12. de Mendonca RM, de Araujo M, Levy CE, et al. Prospective evaluation of HSV, Candida spp., and oral bacteria on the severity of oral mucositis in pediatric acute lymphoblastic leukemia. Support Care Cancer 2012;20:1101-1107.

13. Redding SW. Role of herpes simplex virus reactivation 
in chemotherapy-induced oral mucositis. NCI Monogr 1990:103-105.

14. Epstein JB. Mucositis in the cancer patient and immunosuppressed host. Infect Dis Clin North Am 2007;21:503522.

15. Epstein JB, Ransier A, Sherlock CH, Spinelli JJ, Reece D. Acyclovir prophylaxis of oral herpes virus during bone marrow transplantation. Eur J Cancer B Oral Oncol 1996;32B:158-162.

16. Redding SW, Montgomery MT. Acyclovir prophylaxis for oral herpes simplex virus infection in patients with bone marrow transplants. Oral Surg Oral Med Oral Pathol 1989;67:680-683.

17. Woo SB, Sonis ST, Sonis AL. The role of herpes simplex virus in the development of oral mucositis in bone marrow transplant recipients. Cancer 1990;66:2375-2379.

18. Lalla RV, Bowen J, Barasch A, et al. MASCC/ISOO clinical practice guidelines for the management of mucositis secondary to cancer therapy. Cancer 2014;120:1453-1461.

19. Tomblyn M, Chiller T, Einsele H, et al. Guidelines for preventing infectious complications among hematopoietic cell transplantation recipients: a global perspective. Biol Blood Marrow Transplant 2009;15:1143-1238.

20. Vanderas AP, Gizani S, Papagiannoulis L. Progression of proximal caries in children with different caries indices: a 4-year radiographic study. Eur Arch Paediatr Dent 2006;7:148-152.

21. World Health Organization. WHO Handbook for Reporting Results of Cancer Treatment. Geneva (CH): WHO, 1979 .

22. Stiff PJ, Emmanouilides C, Bensinger WI, et al. Palifermin reduces patient-reported mouth and throat soreness and improves patient functioning in the hematopoietic stem- cell transplantation setting. J Clin Oncol 2006;24:51865193.

23. Bubley GJ, Chapman B, Chapman SK, Crumpacker CS, Schnipper LE. Effect of acyclovir on radiation- and chemotherapy-induced mouth lesions. Antimicrob Agents Chemother 1989;33:862-865.

24. Carrega G, Castagnola E, Canessa A, et al. Herpes simplex virus and oral mucositis in children with cancer. Support Care Cancer 1994;2:266-269.

25. Bergmann OJ, Mogensen SC, Ellegaard J. Herpes simplex virus and intraoral ulcers in immunocompromised patients with haematologic malignancies. Eur J Clin Microbiol Infect Dis 1990;9:184-190.

26. Mendonca RM, Araujo Md, Levy CE, et al. Oral mucositis in pediatric acute lymphoblastic leukemia patients: evaluation of microbiological and hematological factors. Pediatr Hematol Oncol 2015;32:322-330.

27. Freifeld AG, Bow EJ, Sepkowitz KA, et al. Clinical practice guideline for the use of antimicrobial agents in neutropenic patients with cancer: 2010 update by the Infectious Diseases Society of America. Clin Infect Dis 2011;52:427431.

28. Wald A, Huang ML, Carrell D, Selke S, Corey L. Polymerase chain reaction for detection of herpes simplex virus (HSV) DNA on mucosal surfaces: comparison with HSV isolation in cell culture. J Infect Dis 2003;188:13451351.

29. Nagel MA, Choe A, Traktinskiy I, Gilden D. Burning mouth syndrome due to herpes simplex virus type 1. BMJ Case Rep 2015;2015.

30. Lee JT, Kim YT. Prevalence of antibody to herpes simplex virus. Korean J Dermatol 1993;31:38-46. 